\title{
Tension fatigue failure prediction for HMPE fibre ropes
}

\author{
Humeau Corentin ${ }^{1,{ }^{\star}}$, Davies Peter ${ }^{1}$, Smeets P. ${ }^{2}$, Engels T.A.P. ${ }^{3,4}$, Govaert L.E. ${ }^{4}$, Vlasblom M. ${ }^{2}$, \\ Jacquemin Frédéric ${ }^{5}$
}

${ }^{1}$ Laboratoire Comportement des Structures en Mer, IFREMER Centre Bretagne, CS 10070, ZI de la Pointe du Diable, 29280 Plouzané, France

2 DSM Dyneema, Urmonderbaan 22, 6167 Geleen, The Netherlands

${ }^{3}$ DSM Materials Science Center, Urmonderbaan 22, 6167 Geleen, The Netherlands

${ }_{5}^{4}$ Eindhoven University of Technology, 5612 AZ Eindhoven, The Netherlands

${ }^{5}$ EMM-GeM, 58 rue Michel Ange, 44600 Saint-Nazaire, France

* Corresponding author : Corentin Humeau, email address : corentin.humeau@ifremer.fr

peter.davies@ifremer.fr ; frederic.jacquemin@univ-nantes.fr

\begin{abstract}
:
In order to predict the lifetime of fibre rope mooring lines it is essential to be able to predict their behaviour under tension fatigue. Creep failure is known to be a major contributor to fatigue in synthetic fibres and models to predict creep failure are well-established. We show that expansion of such models to varying loading conditions allows the prediction of the fatigue performance. However, it is difficult to design tests to quantify the fatigue performance for HMPE ropes since often premature failure occurs due to external abrasion and viscous heating due to too high testing frequencies or amplitudes. This paper presents a testing methodology which allows tensile fatigue lifetime to be evaluated by testing at higher temperature to avoid premature abrasion failure. We also show that when the temperature evolution due to viscous heating is properly accounted for the modelling framework presented can be effectively used to describe the premature failure occurring due to this heating effect. Results from tests on yarns and small ropes are presented, and a predictive model for rope fatigue lifetime has been validated.
\end{abstract}




\section{Introduction}

The offshore oil and gas and marine renewable energy industries both require innovative mooring techniques in order to extend operational windows of floating structures. In this context, conventional wire rope and chain configurations are limited, both in terms of water depth and dynamic response, due to material and economic considerations. During the last decades, alternative mooring systems, involving synthetic ropes, have been studied and tested, in particular for station-keeping of floating platforms offshore as in studies [1]-[7]. While some of these systems are still at the test stage, others are currently in use.

Among the alternative systems, HMPE (High Modulus PolyEthylene) fibre ropes show great potential, thanks to their high mechanical performance combined with light weight. However, the remaining issue with most HMPE ropes is their poorer creep behaviour. For this reason recent materials developments have focussed on alternative HMPE fibres with much lower creep deformation, such as the Dyneema ${ }^{\circledR}$ DM20 grade, presented in [8]. DSM is the inventor and manufacturer of Ultra High Molecular Weight PolyEthylene (UHMWPE) fiber branded as Dyneema®).

The knowledge on the creep performance of HMPE materials builds to a large extend on the classical work of Wilding and Ward on melt-spun polyethylene fibers, where the main emphasis was on deformation as a function of load rather than on time-to-failure [9]-[12]. Govaert and Lemstra extended the observations to gel-spun fibers [13] which form the basis of conventional HMPE fibres and ropes, and if we look back at previous studies [1]-[3], [8]-[12], [14] and [15], we can conclude that we now have a good understanding of the creep behaviour of HMPE from yarns to ropes which is governed by plastic deformation.

In terms of dynamic failure mechanisms, we can highlight two groups: the crack growth propagation mechanism, which is strongly frequency and cycle time dependent, and the plastic deformation failure, that only depends on the effective time spend under load, as presented in [16]. In the case of HMPE fibres the plastic deformation mechanism is seen to dominate the long term loading performance and to date no observations are known of a transition towards a crack growth or frequency dominated regime. Given the fact that these fibres are highly oriented makes e.g. the crack propagation failure unlikely. For these reasons we expect the tension fatigue failure to be dictated by plastic deformation failure and not by crack propagation.

The aim of the present work is to validate the transposition of the prediction method based on the creep behaviour up to dynamic testing. First, this work will verify this hypothesis at the yarn level. Then, in order to have a better understanding of the real-life case, this study aims to validate this model for small ropes. Before translating the prediction from yarn to ropes we have to identify the critical mechanisms that could be introduced by the construction: external abrasion (friction at end connections), internal heating processes, which are likely to be more critical in ropes than in yarns (due to the larger diameter), and internal abrasion (yarn on yarn friction). Note that these additional failure mechanisms are more apparent in short-term testing protocols that are typically performed at high load and frequency and in most cases will not be relevant for actual applications. 


\section{Background}

\subsection{Synthetic rope fatigue behaviour}

HMPE fibres are relatively new compared to other synthetic ropes such as polyamide or polyester. For common fibres fatigue failures have already been studied in [4], [17]-[19]. Those studies highlighted three types of failure under tension fatigue loading: failures induced by creep phenomena, by external abrasion or due to internal abrasion.

The most common failure for tension fatigue is creep related. In this case we can use models established for static creep strain prediction and replace the constant stress by a cyclic one (see [4], [17], [18]). If the failure is governed by creep, and no other mechanisms interfere, the failure time will be independent of the testing frequency only depend on the time spend under load.

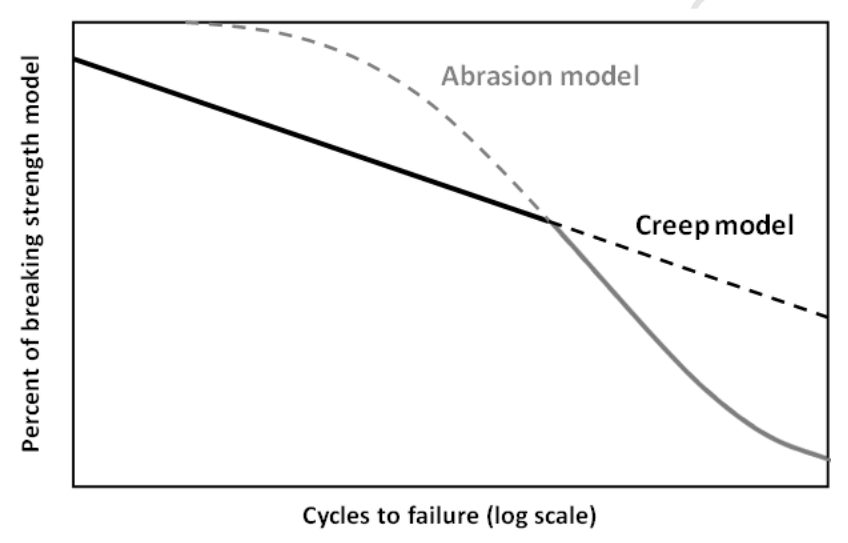

Figure 1.1 Model prediction abrasion vs. creep based on study [17].

Abrasion phenomena can induce deviations from this type of prediction as shown in [17]. In this case, the study of the failure location allows the type of friction involved to be identified. For example, Mandell identified a failure located in the end connection when the experimental results started to deviate from the creep prediction, which oriented the explanation to an external abrasion issue due to the friction at connections (results in Figure 1.1). This type of mechanism only dependents on the number of cycles as shown in [17], and therefore is governed by testing frequency. Bending over sheave tests are more relevant to study this phenomenon.

The work of Mandell also evokes the possible influence of internal abrasion for high numbers of cycles and low loads. Nevertheless they were not able to identify this failure type experimentally on ropes, and suggested that this can be better understood by yarn on yarn testing.

Fatigue investigations on HMPE have been mainly focused on rope bending fatigue performance (e.g. [20]-[24]) as it was identified as the most severe case for handling lines. Nevertheless to be able to quantify the life time expectancy of a rope we have to establish a theory for pure tension fatigue. 


\subsection{Yarn creep behaviour}

Studies on fatigue lifetime prediction for synthetic fibres indicate a good prediction of dynamic fatigue life time based on creep failure data in most cases (see [4], [17]-[19]). Moreover, HMPE is known to show high creep strain which makes this phenomenon even more critical in the case of fatigue loading. For these reasons the first step of fatigue life time prediction is to transpose creep theory to dynamic loading.

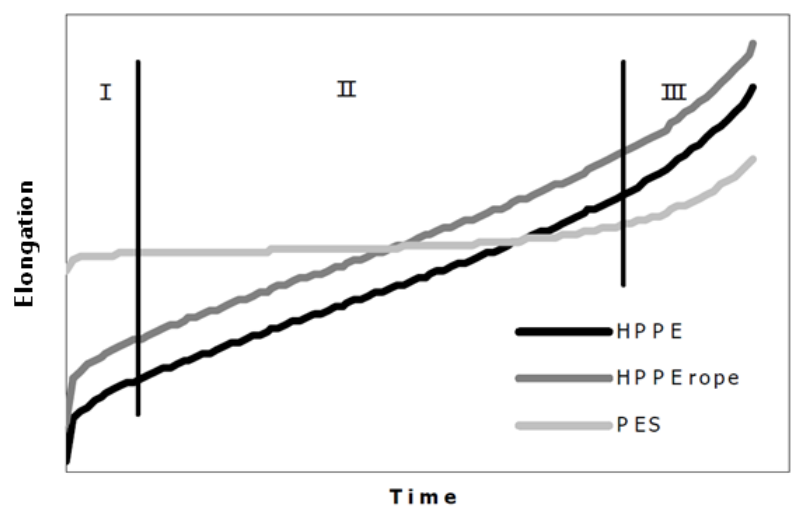

Figure 1.2. Representation of the three regimes of HMPE creep behaviour [2] (NB: HPPE was the previous name of HMPE fibres).

Creep behaviour on Dyneema ${ }^{\circledR}$ fibres is an issue well understood at the yarn level, and it can be described in three parts (shown in Figure 1.2):

- I: The primary creep step represents the delayed elastic strain induced by the stretching of non-crystalline chain segments. This elongation follows a logarithmic law with respect to the time, is proportional to the applied stress and fully reversible if enough time is given for recovery.

- II: The secondary creep step translates the slippage of molecular chains. Strain is proportional to time and can be represented using a power law of the stress (with a power value dependant on the HMPE grade: crystallinity, orientation ...).

- III: The tertiary creep stage begins when the first filament breaks and can be interpreted as a self-amplifying failure mechanism where increasing filament failures and stress amplify each-other, and is absent in case of testing single filaments. It is not modelled due to its high variability from one specimen to another and is governed by filament failure statistics.

A method to predict static creep has been investigated in previous studies [2], [3], [8], [16], [17], [25] and [27] based on the assumption that polymers will break above a constant maximum plastic strain. This method is detailed and modified in the next section for dynamic loading. 


\section{Life time prediction}

\subsection{Theory of yarns}

\subsubsection{General case}

As suggested above, the HMPE yarn life time under dynamic loading is controlled by the creep mechanism. Moreover, studies [25] and [26] have shown that creep failure occurs when plastic strain reaches a critical value: $\varepsilon_{p l-c r i t}$ (independent of the loading case), which can be defined as the integration of strain rate over yarn life time (see Eq. 2.1.)

$$
\varepsilon_{p l-c r i t}=\int_{0}^{t t} \dot{\varepsilon}_{p l-d} d t=\int_{0}^{t t_{f s}} \dot{\varepsilon}_{p l-s} d t
$$

In Eq. 2.1., $\dot{\varepsilon}_{\mathrm{pl}-\mathrm{s}}$ and $\dot{\varepsilon}_{\mathrm{pl}-\mathrm{d}}$ respectively stand for the plastic strain rates under static and dynamic loading. Moreover, $\mathrm{tt}_{\mathrm{fs}}$ and $\mathrm{tt}_{\mathrm{fd}}$ respectively stand for the time to failure in static and dynamic loading.

Studies [16], [17], [27] established expressions for the creep strain rate as a function of temperature and stress. In this study we use the expression from [27] which considers the creep strain rate as a power function of the stress and follows an Arrhenius law of the temperature. The plastic strain rate under cyclic loading can be expressed as in the static case by replacing the constant stress by a dynamic one (see Eq. 2.2.).

$$
\dot{\varepsilon}_{p l-d}(t)=C \times a_{T} \times \sigma(t)^{m}
$$

In Eq. 2.2., $C$ and $m$ are two (material) constants, $\sigma(t)$, the dynamic stress signal and $a_{T}$, a factor following an Arrhenius law of the temperature: $a_{T}=e^{\frac{\Delta U}{R_{g}}\left(\frac{1}{T_{0}}-\frac{1}{T}\right)}$ where $\Delta$ Uis the activation energy, $R_{g}$ the universal gas constant, $T$ the test temperature and $T_{0}$ a reference temperature.

The data base for static creep life time and parameters $\left(C, \Delta U, T_{0}, m\right.$ and $\left.\varepsilon_{p l-c r i t}\right)$ is well developed, which makes it interesting to compare the life time in dynamic and static loading for the same temperatures. To achieve such a comparison, we have to make the assumption that time to failure is quite long compared to the period of the dynamic loading $t_{c y c l e}$, and that we can consider the strain as the integration of the stress function over one cycle multiplied by the number of cycles. Appling this transformation to Eq. 2.1. we obtain a relationship between $t t_{f d}$ and $t t_{f s}$, linked with a dynamic shift factor $a_{s d}$ as written in

Eq. 2.3. The theory behind the dynamic shift factor is developed in [26]. 


$$
t t_{f d}=t t_{f s} \frac{\int_{0}^{t_{c y c l e}} \sigma_{\max }^{m} d t}{\int_{0}^{t_{c y c l e}} \sigma(t)^{m} d t}=\frac{t t_{f s}}{a_{s d}}
$$

With $t t_{f s}=\frac{\varepsilon_{p l-c r i t}}{C \times a_{T} \times \sigma_{\max }{ }^{m}}$ and $a_{s d}=\frac{\int_{0}^{t_{c y c l e}} \sigma(t)^{m} d t}{t_{c y c l e} \times \sigma_{\max }{ }^{m}}$

To go further in the dynamic shift factor calculation, we have to apply a specific function for dynamic stress.

\subsubsection{Sinusoidal case}

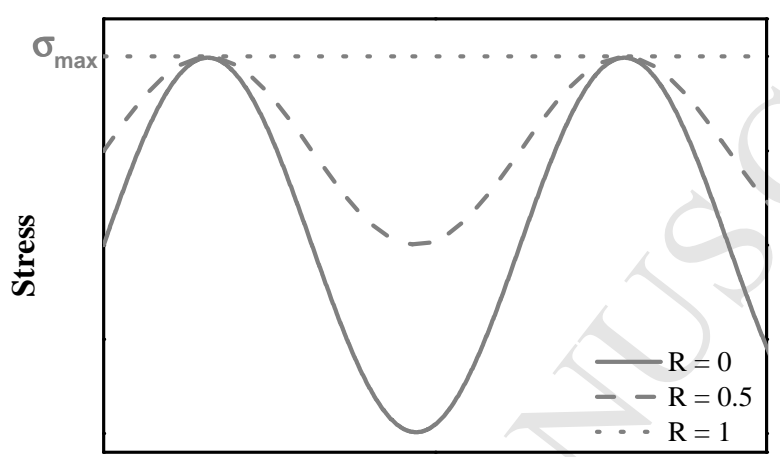

Time

Figure 2.1: Sinusoidal stress versus time for different $R$ ratio

The dynamic load is chosen as a sinusoidal function of time. There are three main methods to express sinusoidal stress, (1) either with the maximum and minimum stresses, (2) with mean and amplitude, or (3) with maximum stress and the stress ratio, $R=\sigma_{\min } / \sigma_{\max }$. In our case we want to compare the static creep test with the fatigue response, so the use of the $\mathrm{R}$ ratio allows us to represent the creep test as a fatigue test with $=1$ (see Figure 2.1). Then, the sinusoidal stress expression can be introduced in the previous equations Eq. 2.2. to get the dynamic creep strain rate, as shown in Eq. 2.4.

$$
\varepsilon_{p l-c r i t}(t)=C \times a_{T} \times\left[\left(\sigma_{\max } \frac{1+R}{2}\right)+\left(\sigma_{\max } \frac{1-R}{2}\right) \sin (t \omega)\right]^{m}
$$

With $\omega=2 \pi / t_{\text {cycle }}$ the frequency

The dynamic shift factor expression can then be simplified, using the variable $t^{\prime}=t^{t} / t_{\text {cycle }}$ and the integration of the parameter $\varepsilon_{p l \text {-crit }}$ gives Eq. 2.5., presented hereafter.

$a_{s d}=\frac{\int_{0}^{t_{c y c l e}}\left(\frac{1+R}{2}+\frac{1-R}{2} \sin (t . \omega)\right)^{m} d t}{\sigma_{\max } m}=t_{\text {cycle }} \int_{0}^{1}\left(\frac{1+R}{2}+\frac{1-R}{2} \sin \left(2 \pi . t^{\prime}\right)\right)^{m} d t^{\prime}$ 
The expression in Eq. 2.5. for dynamic shift factor from static to dynamic highlights a time to failure independent of frequency values. For a given fibre type, $a_{s d}$ is dependents on one parameter related to the loading condition: the $\mathrm{R}$ ratio, which induces variations of time to failure inverse to variations of the $\mathrm{R}$ ratio (see Figure 2.2), and one material dependent parameter, $\mathrm{m}$. To conclude, the prediction curve from static to dynamic tests is only shifted along the time to failure axis.
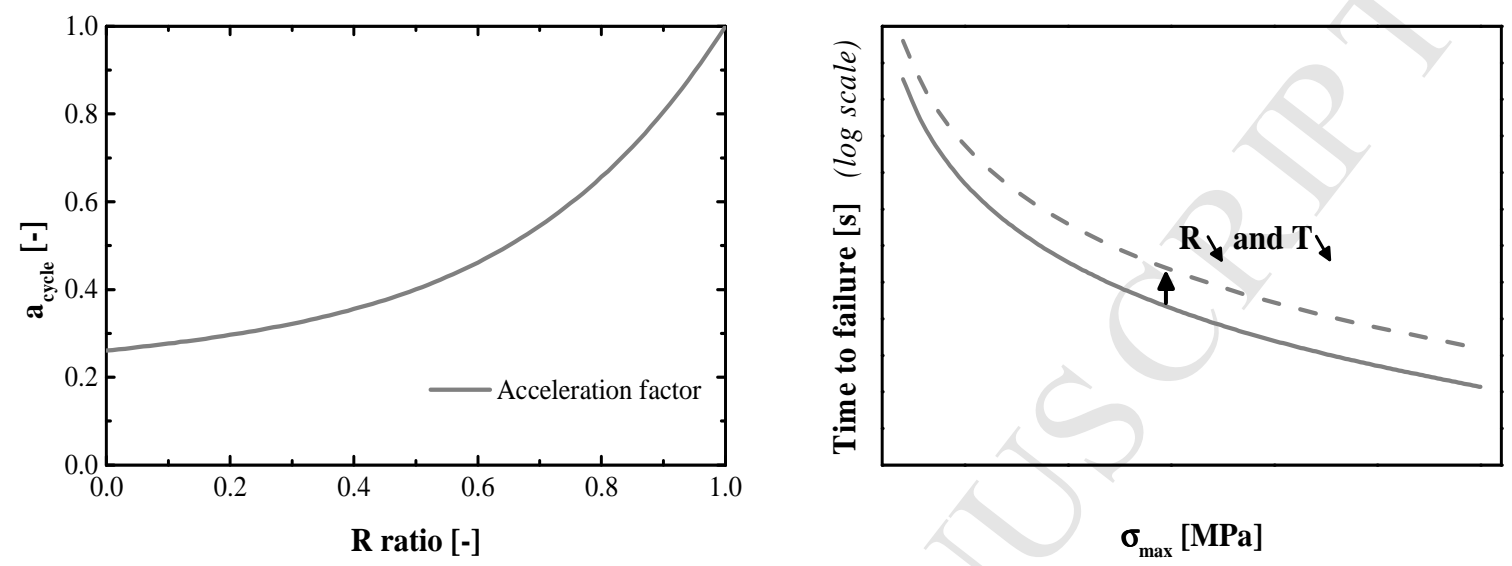

Figure 2.2: Influence of $R$ on dynamic shift factor for Dyneema ${ }^{\circledR} S K 78$ (for $m=4.43$ ) [left] and $R$ and temperature on dynamic time to failure prediction [right]

\subsubsection{Time-Temperature superposition}

To avoid end termination effects and to get a failure in the central part of the rope sample which is loaded in tension, the tests can be performed at high temperature, to accelerate the plastic failure phenomenon. Results obtained at high (testing) temperatures can be translated to the relevant low application temperatures using. the time-temperature equivalence method.

As noted above, the creep strain rate is temperature dependent as shown in Eq. 2.2. This temperature dependence is governed by an Arrhenius equation and this same temperature dependence can be used for times-to-failure measured at different temperatures under the same loading condition: A straightforward relationship between times-to-failure involving only a Arrhenius temperature shift factor $\left(a_{T_{1-2}}\right)$ is then obtained as shown in Figure 2.2 and Eq. 2.6.

$$
t t_{f}\left(T_{1}\right)=t t_{f}\left(T_{2}\right) e^{\frac{\Delta U}{R_{g}}\left(\frac{1}{T_{1}}-\frac{1}{T_{2}}\right)}=t t_{f}\left(T_{2}\right) \cdot a_{T_{1-2}}
$$

Eq. 2.6.

For a given fixed temperature this temperature shift factor is constant, which means that a master curve can be created by a simple translation (shift) of the results along the time axis for each temperature (see Figure 2.2). 


\section{2. $\quad$ Rope theory}

In order to sufficiently capture the complexity of the final application it is important to consider the rope scale. The construction chosen is a braided rope made of 12 twisted strands each of 4 yarns of 1760 dtex Dyneema® SK78 HMPE fibres.

For ropes subjected to cyclic loading we can apply the same theory as for yarns by taking into account the angle of filaments in the ropes to calculate the stress in the yarns. Previous studies established simple analytical relationships between load applied to twisted ropes and stress in the filament (as [28], [29]), but in our case the construction is a braid, for which there is no simple theory (see [30]). Another way to estimate the stress in yarns is to take the average braid angle $\left(\alpha=24^{\circ}\right)$ and consider that all the filaments have this same angle with respect to the loading direction (see Figure 2.3) and that the rope angle is not changing significantly during testing. From this assumption we can find the stress applied on the yarns considering that the load on rope is the projection of the load on yarn over the loading direction (see Eq. 2.7.)

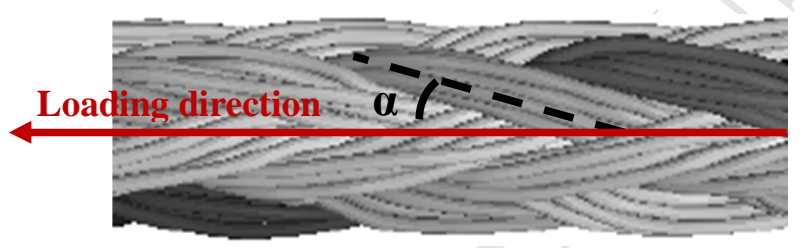

Figure 2.3: Representation of the average angle of filaments $(\alpha)$ to the loading direction, defined to be equal to the braided angle.

$$
F_{\text {yarn }}(t)=\frac{F_{\text {rope }}(t)}{\cos (\alpha)}
$$

The rope construction also induces higher friction effects between the yarns and the terminations, this can generate premature failure in some cases such as at high frequencies, amplitudes or loads. Finally, increased diameter ropes lead to higher temperatures than within a single yarn, since the heat created by plastic deformation diffuses slower than in yarns. The friction phenomena can also increase the heating process. These issues must be studied before testing ropes, to define the range of the study and to avoid unrealistic heating or abrasion effects.

\section{Experimental results}

\subsection{Yarn behaviour from previous works}

First this model has been verified on Dyneema ${ }^{\circledR}$ SK75 yarn samples in the study [31]. The most frequently used linear density for this material is around 1760dtex for yarns but other linear densities are available, from 110 dtex to 2640 dtex, [32]), 


\subsubsection{Material}

To be able to compare dynamic loading to the ultimate static strength, static tensile tests were performed before investigating the tension fatigue failure. Tests were performed following the ASTM D885 method. Concerning sample preparation and conditioning, yarn bobbins were conditioned at a temperature of $21^{ \pm 1}{ }^{\circ} \mathrm{C}$ and relative humidity of $60^{ \pm 10} \%$ for at least two hours before testing, and each sample was twisted with 40 turns/meter. The tensile testing was performed with a constant extension rate of $250 \mathrm{~mm} / \mathrm{min}$, a gage length of $500 \mathrm{~mm}$, a load cell of maximum force of $2 \mathrm{kN}$ and a pretension of $0.2 \mathrm{cN} /$ tex. Moreover clamps were used that were designed to minimize the stress concentration.

From these tensile tests, we can get material properties as: the maximum force (in $\mathrm{N}$ ) and the elongation determined as the gage displacement over the initial gage length (in $\mathrm{mm} / \mathrm{mm}$ ). These properties are then combined with the sample linear density and volume weight to obtain the maximum stress and the modulus (both in GPa). For the Dyneema ${ }^{\circledR}$ SK75, we get a tenacity of $3.6 \mathrm{GPa}$ and a modulus around $110 \mathrm{GPa}$

\subsubsection{Dynamic behaviour}

The static and dynamic results obtained in study [31] show a good correlation between the model prediction and experimental data. There is only a slight deviation which can be highlighted at high stress, which was shown to be induced by viscous heating in the material by varying the test frequency. Parameters used for the model are listed in table 1 below:

\begin{tabular}{ccccc} 
& $\mathrm{C}^{*}$ & $\mathrm{~m}$ & $\Delta \mathrm{U}$ & $\varepsilon_{\text {cr }}$ \\
& {$[-]$} & {$[-]$} & {$[\mathrm{kJ} / \mathrm{mol}]$} & {$[-]$} \\
\hline SK75 & 0.14 & 3.58 & 108 & 0.26 \\
SK78 & 0.00032 & 4.43 & 112 & 0.26 \\
\hline \multicolumn{5}{c}{ for a reference temperature $\left(\mathrm{T}_{0}\right)$ of $70^{\circ} \mathrm{C}$}
\end{tabular}

Table 1: model parameters for Dyneema® ${ }^{\circledR}$ SK75 and SK78 yarns.

This good agreement between prediction and experiment compelled to perform the same tests on rope scale.

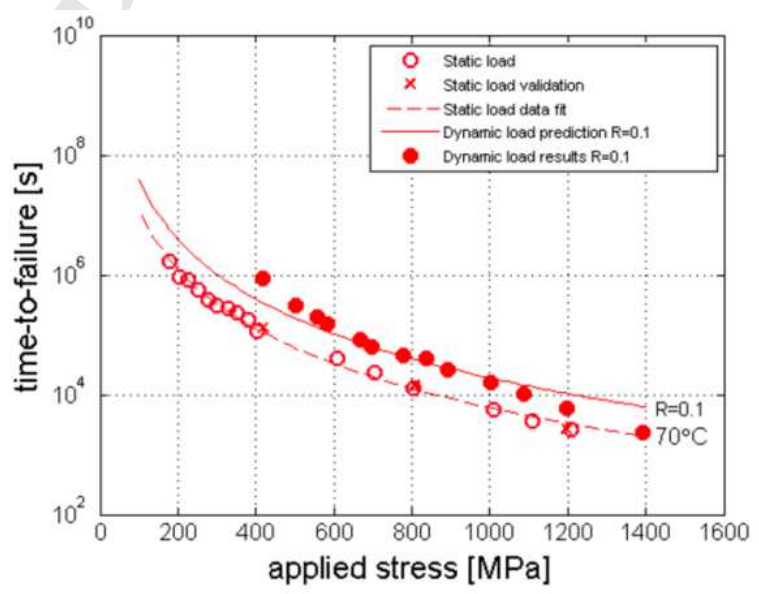

Figure 3.1. Time-to-failure at $70^{\circ} \mathrm{C}$ for Dyneema ${ }^{\circledR}$ SK75 yarns under static and dynamic loading obtained by model prediction [lines] and by experimental testing [points] (from [31]). 


\subsection{Rope behaviour}

At the rope level the tests were performed on a fibre with higher creep resistant properties, the Dyneema ${ }^{\circledR}$ SK78, with a linear density of 1760 dtex. Ropes typically allow the measurement of mechanical properties with less variability. The rope construction used in this work has a 12x1 braided construction with a total linear density of $9.1 \mathrm{~g} / \mathrm{m}$ and substrands composed of 4 times assembled yarns of $4 \times 1760$ dtex.

\section{Material}

Before investigating the behaviour of the rope in tension fatigue, static tensile tests were performed to determine the reference mechanical properties. The rope was tested with a special set-up: samples are maintained in position and wound three times over a cylinder with a large enough diameter to sufficiently lower contact pressures and with a specific roughness to prevent slippage and excessive abrasion. This test provides the failure stress of the rope, the strain (and therefore modulus) can however not be determined accurately since some slippage of the rope on the clamps cannot be avoided. Strength values for the ropes are listed in Table 2.

\begin{tabular}{rrr} 
Sample & $\begin{array}{r}\text { Tenacity } \\
{[\mathbf{c N} / \text { dtex] }}\end{array}$ & $\begin{array}{r}\text { Stress } \\
{[\mathbf{G P a}]}\end{array}$ \\
\hline $\mathbf{1}$ & 20.24 & 1.96 \\
$\mathbf{2}$ & 21.05 & 2.04 \\
$\mathbf{3}$ & 21.77 & 2.11 \\
$\mathbf{4}$ & 20.01 & 1.94 \\
\hline Average & 20.77 & 2.01
\end{tabular}

Table 2: Ultimate stress and tenacity of Dyneema ${ }^{\circledR}$ SK78 ropes

Dyneema ${ }^{\circledR}$ SK78 yarns have a significantly lower creep rate compared to Dyneema ${ }^{\circledR}$ SK75. For this reason the creep life time of Dyneema ${ }^{\circledR}$ SK78 yarns have been measured and modelled over a large range of temperatures, as shown in Figure 3.2. Using Eq. 2.3. and Eq. 2.7. we will be able to predict the time to failure of ropes subjected to fatigue or static loading, in the case of creep dominant failure. Model parameters are listed in Table 1.

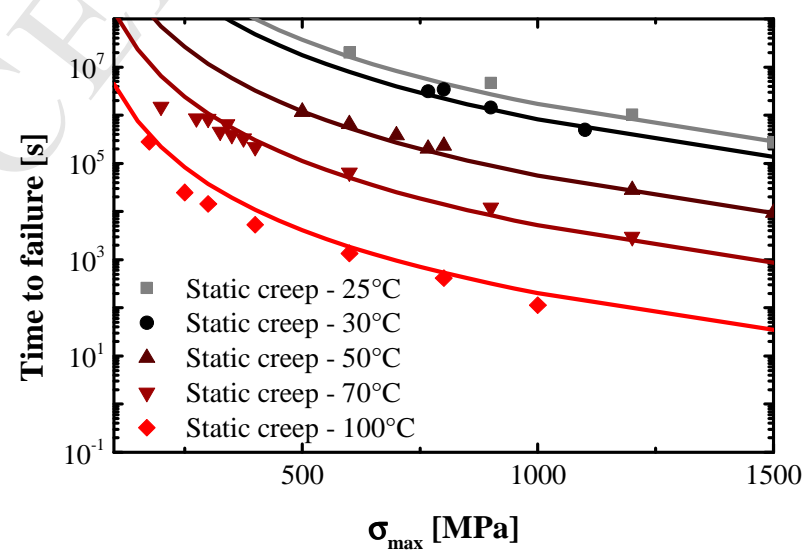

Figure 3.2: Creep time to failure identified (lines) from experimental data (points) on yarns $\left(\mathrm{at} 25,30,50,70\right.$ and $\left.100^{\circ} \mathrm{C}\right)$ 


\subsubsection{Scope of the study}

As stated before the aim of the current investigation is to develop a tool that allows us to predict the failure time of HMPE yarns and ropes under pure tensile fatigue conditions. Clamping of yarns and ropes almost always introduce the risk of failure on the clamps due to external abrasion and the visco-elastic nature of the material introduces the risk of viscous heating due to the cyclic loading. Both can interfere with and obscure test results and need to be studied first.

\section{External abrasion}

While testing ropes in fatigue we have to eliminate the possible failures induced by external abrasion on the clamps. Once this phenomenon is known and controlled, we can define relevant parameters for the study (frequency, temperature, maximum stress).

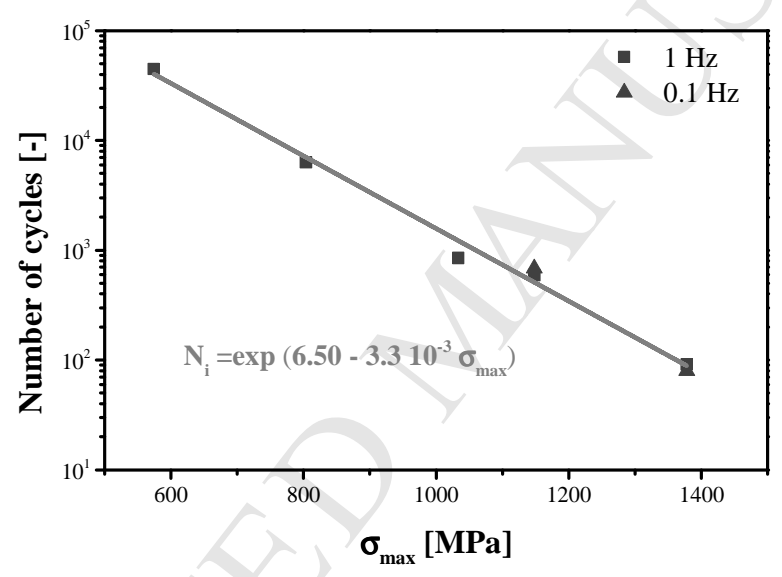

Figure 3.3: S-N curve in case of failure on clamps (external abrasion) for different frequencies at $23^{\circ} \mathrm{C}$

To investigate this aspect, tests were performed at room temperature $\left(23^{\circ} \mathrm{C}\right)$ to increase the creep time to failure without affecting abrasion. Moreover, to check that this is a real cycle dependent phenomenon these tests were performed at two different frequencies.

During these tests it was also verified that failures were indeed the result of an external abrasion (failures near clamps).

Results are summarized in Figure 3.3 and show two main characteristics of external abrasion failures:

- The failure induced by abrasion is entirely dictated by the number of cycles (no influence of the frequency)

- The number of cycles to failure follows an exponential function of the maximum stress applied. 


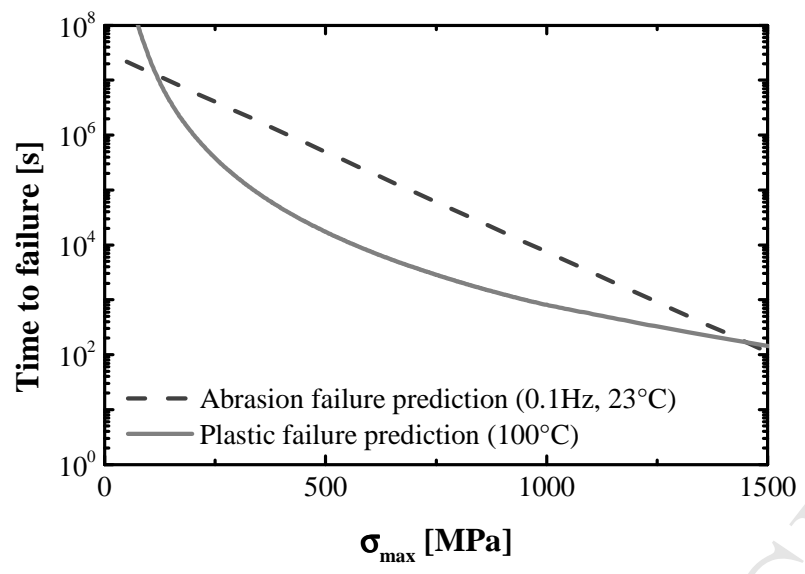

Figure 3.4: Prediction of external abrasion failure compared to prediction of time to failure in creep mode at ${ }^{100^{\circ}} \mathrm{C}$

In this work we want to characterize the creep failure mode in fatigue. To do so we have to avoid any abrasion failure by reducing cyclic damage (low frequency fatigue) and increasing creep rates (high temperature testing). From the experimental results of abrasion failure (shown in Figure 3.3) we established a lifetime prediction model for any frequency. Then, creep dominant failure on Dyneema ${ }^{\circledR}$ SK78 ropes can be predicted from the yarn creep behaviour (see part 3.1) at different temperatures. The comparison between this failure model allows to determine the temperature and frequency of the fatigue tests to obtain a creep failure mode $\left(\mathrm{T}=100^{\circ} \mathrm{C}\right.$ and $\mathrm{f}=0.1 \mathrm{~Hz}$ see Figure 3.4). The frequency of $0.1 \mathrm{~Hz}$ is a realistic parameter which represents a wave period of $10 \mathrm{~s}$ and the temperature of $100^{\circ} \mathrm{C}$ permits us to do tests on an experimentally feasible timescale but avoid approaching the melting range of the material $\left(\mathrm{T}_{\mathrm{m}}=144-152^{\circ} \mathrm{C}\right)$ too closely. Moreover, it is preferred to be able to perform tests at stresses up to 1500 MPa (75\% of ultimate stress). Higher loads would not be interesting since ropes in practical use are usually stressed in the range below 400 to $600 \mathrm{MPa}$.

\section{Heating process}

The second issue is the hysteretic loop created by the viscoelastic behaviour of HMPE. These loops translate energy dissipation during each fatigue loading cycle, and for a cyclically loaded material the main way to lose energy is by heat generation (see Figure 3.5). Heating can have two negative effects on experimental results: the temperature rise will induce a shift in creep rates and failure times as Eq. 2.6. indicates, and for tests performed close to the fusion temperature of HMPE, the heating can induce a melting process inside the sample. In the case of rope testing, we assume that heating is mainly generated by viscoelastic effects, especially for high loads, frequency and amplitude. Moreover, this phenomenon is highly dependant on the convection heat loss to the surroundings and will therefore depend strongly on the frequency of testing and convective surrounding (environmental temperature) of the sample.

Therefore, the following experiments aims to have been performed on rope samples and the temperature within has been recorded using a thermocouple (type $\mathrm{K}$ ) with a recording period equal to at least three fatigues cycles to reduce the noise induced by the cycles. 


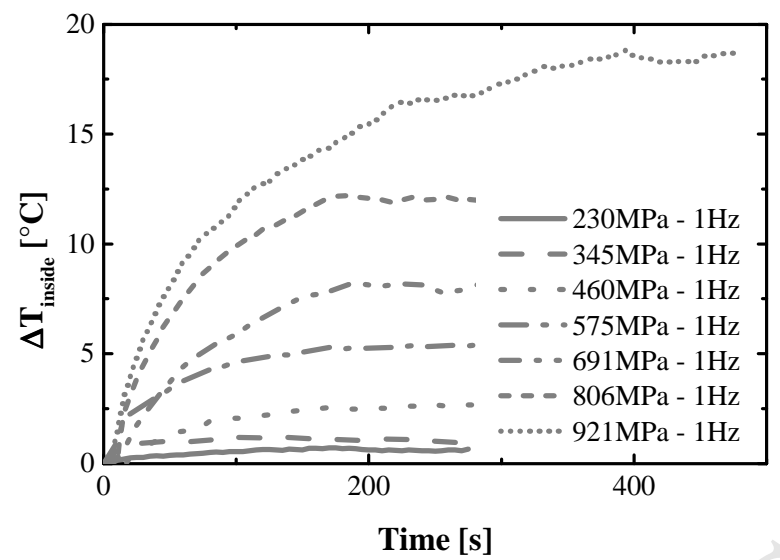

Figure 3.5: Temperature rise inside the rope vs. time for different maximal strength applied at $23^{\circ} \mathrm{C}$

Based on these observations we developed an empirical model which can predict the heating process inside the rope using simple mathematical laws. For this study we do not consider the transient state, only the maximum temperature value inside the rope (Figure 3.6).

Heating of the ropes depends on the maximum stress applied $\sigma$, the surrounding temperature, $T$, the frequency, $f$, and the load ratio, $R$. To identify the influence of these parameters on heating, each of them was successively considered as a variable while the others were kept constant (Figure 3.6) which provides an expression of the heating depending on the variable parameter. In this case we assume that the influence of each parameter on heating is independent of the others.

The observation of temperature variations allowed an exponential dependence on maximum applied stress to be identified (Figure 3.6 [top left]). Moreover we know that when the stress is equal to zero, no heating will take place, so we obtain the following expression:

$$
\Delta T_{\max }=\left(e^{\sigma_{\max } / \sigma_{0}}-1\right) \cdot g(T, f, R)
$$

With $g$ a function of environmental temperature (T), frequency (f) and $\mathrm{R}$ ratio; $\sigma_{\max }$ the maximum stress applied; $\sigma_{0}$ a constant proportional to a stress and equal to $357 \mathrm{MPa}$ for this case.

The frequency effects have been studied for four different stresses, represented in Figure 3.6 [top right], and in each case it highlights a linear dependence of temperature rise on frequency. Moreover, the heating process will disappear for a frequency equal to zero (case of the static creep loading). These two observations led to the development of a heating process expression, from Eq. 3.1. to:

$$
\Delta T_{\text {inside }}=\left(e^{\sigma_{\max } / \sigma_{0}}-1\right) \cdot f \cdot h(T, R)
$$

With $\mathrm{h}$ a function of $\mathrm{T}$ and $\mathrm{R}$. For a frequency of $0.1 \mathrm{~Hz}$, the heating process can therefore be neglected for the stress range here (up to $1500 \mathrm{MPa}$ ).

Finally the heating process is dependent on the surrounding temperature. To identify its influence on the temperature rise within the rope we conducted tests under different conditions. Results summarised in Figure 3.6 [bottom] show a linear dependency of the heating process with the surrounding temperature, 
and for temperatures below $0^{\circ} \mathrm{C}$ there is no heating phenomenon, which modifies Eq. 3.2. in Eq. 3.3., considering $k(R)$ as a function of $\mathrm{R}$ ratio, equal to $7.10^{-2} \mathrm{~s} .{ }^{\circ} \mathrm{C}$ for $\mathrm{R}=0.1$.

$$
\Delta T_{\max }=\left(e^{\sigma_{\max } / \sigma_{0}}-1\right) \cdot T \cdot f \cdot k(R)
$$

This empirical law (Eq. 3.3.) provides a tool to determine if the heating process will be significant based on the testing conditions. Nevertheless the equation has been established for a specific HMPE grade and rope construction (braid angle, dtex, diameter, coating) it should not be applied directly in a case with different parameter(s).
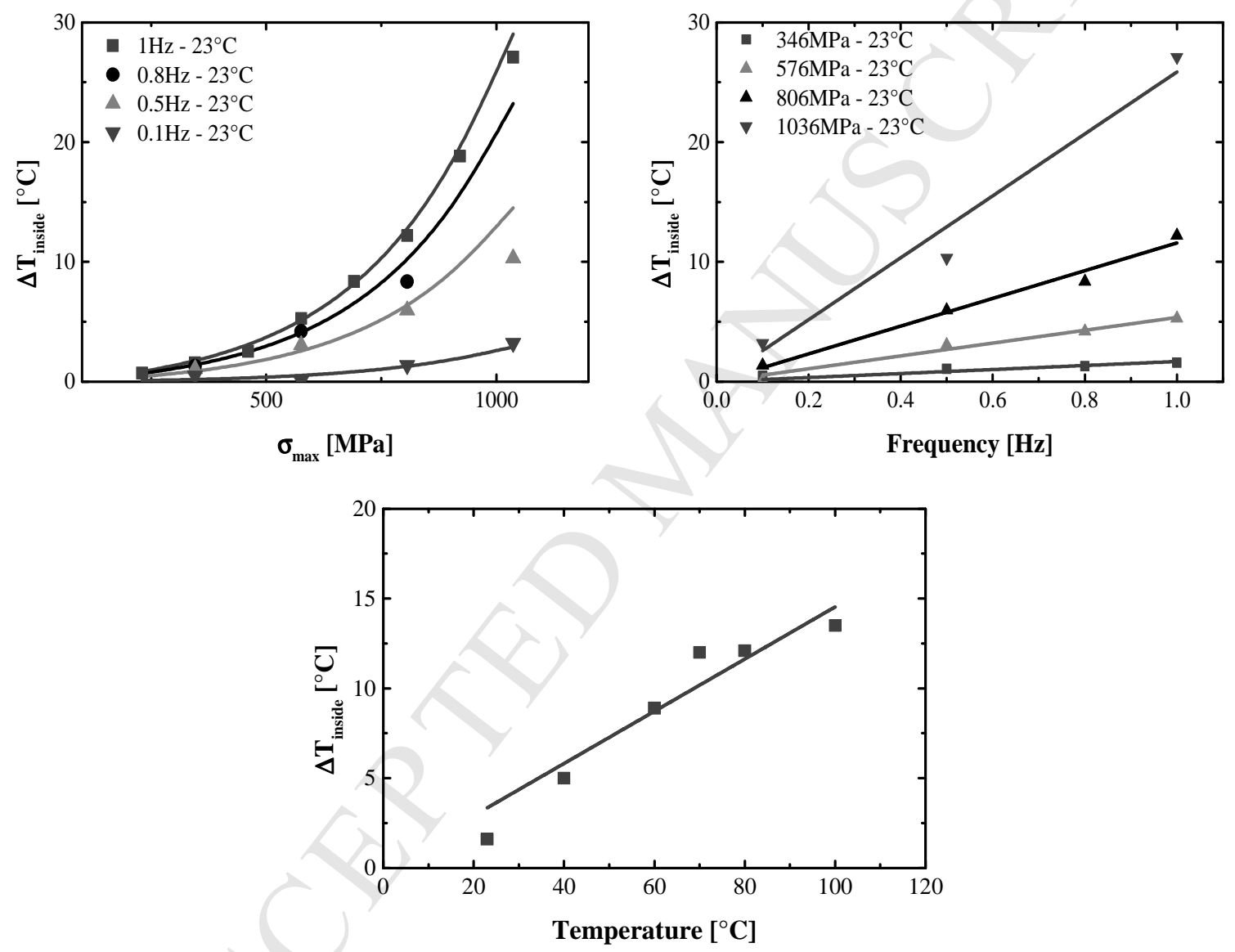

Figure 3.6: Maximum rise of temperature vs. stress applied for different frequencies $\left(\mathrm{R}=0.1, \mathrm{~T}=\mathbf{2 3}{ }^{\circ} \mathrm{C}\right)$ [top left]. Maximum temperature rise as a function of frequency for diffrent stresses $\left(R=0.1, T=23^{\circ} \mathrm{C}\right)$ [top right]. Maximum temperature rise as a function of the environmental temperature $(R=0.1, f=1 \mathrm{~Hz}, \sigma=345 \mathrm{MPa})$ [bottom] 


\subsubsection{Static and dynamic behaviour}

\section{Static creep tests}

The model has been established on yarn experiments, for which the constants have been determined, see table 1. Here we investigate the rope time to failure and compare it to the prediction based on yarn results, but to do so we have to know the stress applied on the yarn during a creep test on the rope. This stress has been determined considering the braided angle as constant and using a simple projection on the yarn axis we obtain an evaluation of its value from Eq. 2.7. This leads to the results and prediction shown in Figure 3.7.

The figure above shows a good correlation between prediction of the time to failure under static creep (based on the yarn results) and experimental data on ropes.

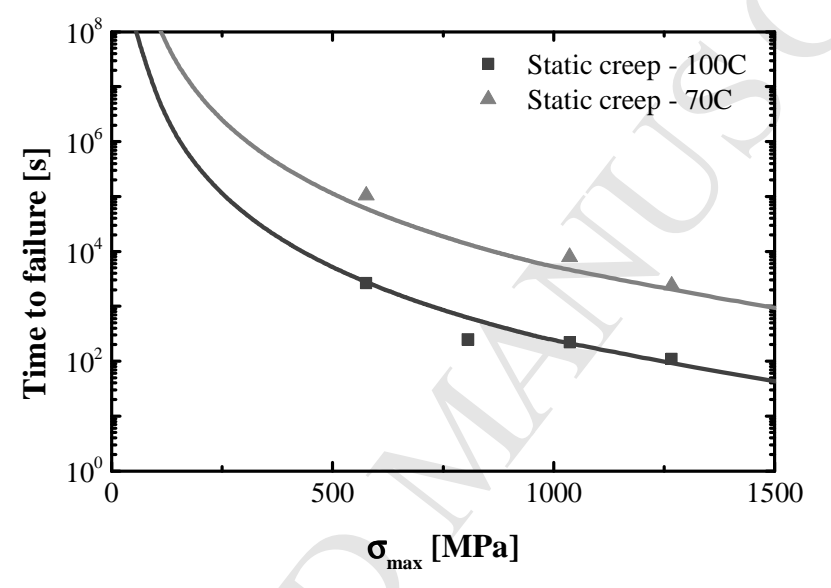

Figure 3.7: Creep time to failure prediction compared to experimental data on ropes $\left(\right.$ at 70 and $\left.100^{\circ} \mathrm{C}\right)$

\section{Dynamic fatigue tests}

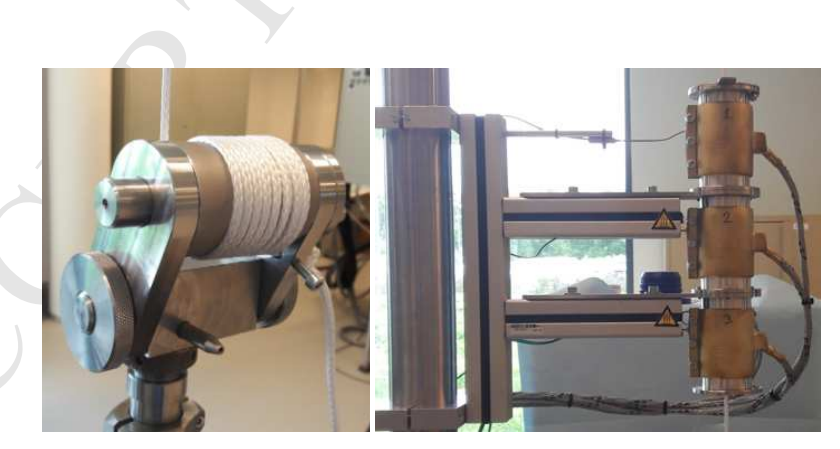

Figure 3.8: Experimental set-ups for dynamic test at high temperature

\section{$\underline{\text { At } 100^{\circ} \mathrm{C}, 0.1 \mathrm{~Hz} \text { and } \mathrm{R}=0.1 \text { : }}$}

It is now possible to define the dynamic test conditions. In order to make the dynamic experiments realistic the frequency was chosen to be close to the wave period, around 10 seconds $(0.1 \mathrm{~Hz}$ frequency) and to avoid external abrasion failure it was necessary to test at $100^{\circ} \mathrm{C}$. The stress ratio has 
been chosen to be equal to 0.1 to simulate a worst case scenario in terms of loading. Figure 3.8 shows the experimental set-up for the tests: the clamps and oven.

Figure 3.9 shows the test results and the prediction (from Eq. 2.3). The prediction is quite accurate up to $900 \mathrm{MPa}$ under these conditions (black dots, Figure 3.9). Above this value the experimental data deviate from the prediction (grey points, Figure 3.9). Boleij already noticed this deviation for the case of yarns in [31]; it could be explained by heating. In part II.2 we established the dependence of temperature rise with temperature, frequency and stress variation. If we insert this simple relation in the predictive model (Eq. 3.4.) we can predict this deviation (Figure 3.9 -right-).

$$
a_{T}=e^{\frac{\Delta U}{R g}\left(\frac{1}{T_{0}}-\frac{1}{T+\Delta T}\right)}=e^{\frac{\Delta U}{R_{g}}\left(\frac{1}{T_{0}}-\frac{1}{T+\left(e^{\sigma_{\max } / \sigma_{0}}-1\right) \cdot T \cdot f \cdot k(R)}\right)}
$$

Eq. 3.4.
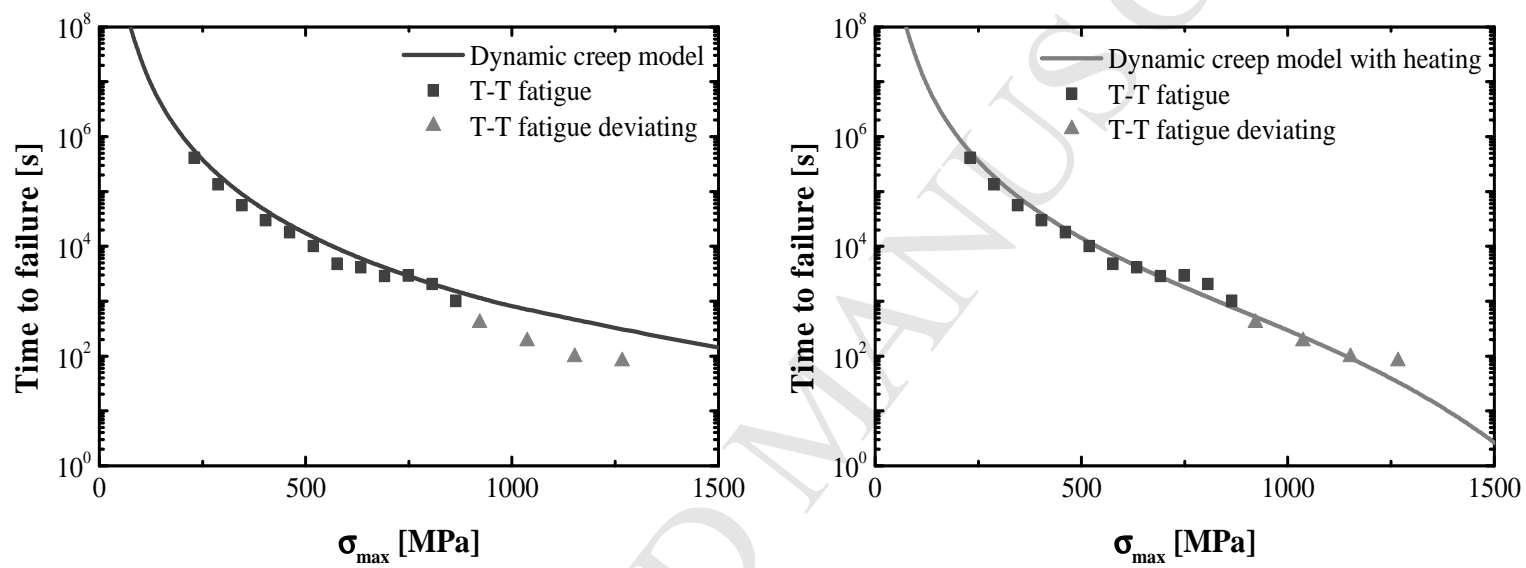

Figure 3.9: Dynamic time to failure prediction compared to experimental data (at $100^{\circ} \mathrm{C}, \mathrm{R}=0.1$ and $\left.0.1 \mathrm{~Hz}\right)$ with out (left) and with (right) heating process taken into account

\section{At $100^{\circ} \mathrm{C}, 0.1 \mathrm{~Hz}$ and $\mathrm{R}=0.5$ :}

The choice to take an $\mathrm{R}$ ratio of 0.1 was made because of the need to investigate the reliability of our model in the case of extreme dynamic loading. Nevertheless marine applications amplitude rarely reach such values; mostly $\mathrm{R}$ ratios are included between 0.3 and 0.6 . To test the robustness of the model on $\mathrm{R}$ variation we performed tests at $\mathrm{R}=0.5$ (within the application range).

$\mathrm{R}$ ratio induces a change on the dynamic shift factor as shown in Eq. 2.5 which will induce a shifting of the failure curve parallel to the time to failure axis. In this case, for an $\mathrm{R}$ ratio of 0.5 the dynamic shift factor is equal to 0.4 and induces a time to failure equal to three quarters of the time to failure of an $\mathrm{R}$ ratio of 0.1. Experimental results and model predictions are summarised in Figure 3.10 . 


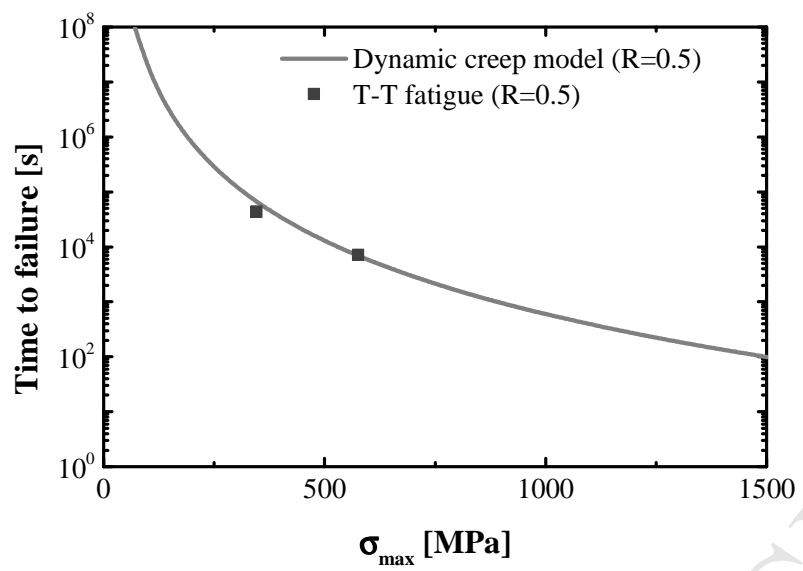

Figure 3.10: Time to failure vs. maximum stress applied for an $\mathrm{R}$ ratio of $0.5\left(100^{\circ} \mathrm{C}\right.$ and $\left.0.1 \mathrm{~Hz}\right)$

The experimental data agree well with the model prediction for the presented case, demonstrating the reliability of the model for different stress ratios. The model was also verified for the static creep case which can be compared to the dynamic case with a frequency equal to zero. In further studies, it would be interesting to validate the model at a different frequency value (other than $0.1 \mathrm{~Hz}$ ).

It has to be noted that some internal abrasion effects have been identified for other fibres (polyester), for which it was assumed that this abrasion affects the time to failure by reducing the cross-sectional area of the rope, a phenomenon dependant on the number of cycles. The problem is that this phenomenon is likely not, or less, affected by the temperature, and could be 'hidden' in the case of our tests, since we used time-temperature superposition to obtain acceptable failure times. It is therefore important to investigate this phenomenon further, in order to understand if there is an internal abrasion mechanism active in case of HMPE ropes, and if so, predict the potential failure due to internal abrasion.

\section{Conclusion}

The results from this study show that the life time for failure under dynamic tension fatigue on HMPE ropes can be successfully predicted based on proper knowledge of yarn creep performance. . To do so, the well-established models for failure under static loading conditions need to be modified to account for dynamic loads. This results in the definition of a dynamic shift factor, only depending on the load ratio, $R$, and material parameter $m$, that effectively results in a shift towards longer failure times in case of comparing the dynamic loading case to the static load case at the maximum load of the dynamic signal.

It is also shown that the model can, in case of loading scenarios in which viscous heating results in increasing temperatures during the test, predict failure times accurately, when the temperature development is taken into account. To this end an empirical temperature model is employed in this study.

Key in developing the model and creating relevant observations was the development of the testing methodology presented in this study. By locally heating the ropes we enabled measuring rope failure times unaffected by external abrasion on the end connections. 
This model still has some limitations: we have to investigate the self heating process, since it can have an influence on the life time, especially in the case of high temperature applications. The temperature inside the rope should never exceed $110^{\circ} \mathrm{C}$ otherwise the constants which depend on the material have to be modified. Finally the study has highlighted failure induced by external abrasion at room temperature and a reduced life time; this shows the importance of the end connection choice for real applications. 


\section{References}

[1] H. S. da Costa Mattos and F. E. G. Chimisso, "Modelling creep tests in HMPE fibres used in ultradeep-sea mooring ropes," Int. J. Solids Struct., vol. 48, no. 1, pp. 144-152, Jan. 2011.

[2] P. Smeets, M. Jacobs, and M. Mertens, "Creep as a design tool for HMPE ropes in long term marine and offshore applications," in MTS/IEEE Conference and Exhibition OCEANS, 2001, 2001, vol. 2, pp. 685-690 vol.2.

[3] M. Vlasblom, J. Boesten, S. Leite, P. Davies, and others, "Development of HMPE fiber for permanent deepwater offshore mooring," in Offshore Technology Conference, 2012.

[4] K. H. Lo, H. Xü, and L. A. Skogsberg, "Polyester Rope Mooring Design Considerations," presented at the The Ninth International Offshore and Polar Engineering Conference, 1999.

[5] P. Davies, M. François, F. Grosjean, P. Baron, K. Salomon, and D. Trassoudaine, "Synthetic Mooring Lines for Depths to 3000 Meters," presented at the Offshore Technology Conference, 2002.

[6] J. F. Flory, S. J. Banfield, C. Berryman, and others, "Polyester mooring lines on platforms and MODUs in deep water," in Offshore Technology Conference, 2007.

[7] S. Banfield, T. Versavel, R. O. Snell, R. V. Ahilan, and others, "Fatigue curves for polyester moorings-a state-of-the-art review," in Offshore Technology Conference, 2000.

[8] M. Vlasblom, J. Boesten, S. Leite, and P. Davies, "Creep and stiffness of HMPE fiber for permanent deepwater offshore mooring," in 2012 Oceans-Yeosu, 2012, pp. 1-7.

[9] M. A. Wilding and I. M. Ward, "Tensile creep and recovery in ultra-high modulus linear polyethylenes," Polymer, vol. 19, no. 8, pp. 969-976, Aug. 1978.

[10] M. A. Wilding and I. M. Ward, "Creep and recovery of ultra high modulus polyethylene," Polymer, vol. 22, no. 7, pp. 870-876, Jul. 1981.

[11] M. A. Wilding and I. M. Ward, "Creep and stress-relaxation in ultra-high modulus linear polyethylene," J. Mater. Sci., vol. 19, no. 2, pp. 629-636, Feb. 1984.

[12] I. M. Ward and M. A. Wilding, "Creep behavior of ultrahigh-modulus polyethylene: Influence of draw ratio and polymer composition," J. Polym. Sci. Polym. Phys. Ed., vol. 22, no. 4, pp. 561-575, Apr. 1984.

[13] L. E. Govaert and P. J. Lemstra, "Deformation behavior of oriented UHMW-PE fibers," Colloid Polym. Sci., vol. 270, no. 5, pp. 455-464, May 1992.

[14] M. J. N. Jacobs, "Creep of gel-spun polyethylene fibres: Improvements by impregnation and crosslinking," PhD thesis, Eindhoven University, 1999.

[15] M. P. Vlasblom and R. L. M. Bosman, "Predicting the Creep Lifetime of HMPE Mooring Rope Applications," in OCEANS 2006, 2006, pp. 1-10.

[16] M. J. W. Kanters, T. Kurokawa, and L. E. Govaert, "Competition between plasticity-controlled and crack-growth controlled failure in static and cyclic fatigue of thermoplastic polymer systems," Polym. Test., vol. 50, pp. 101-110, Apr. 2016.

[17] J. F. Mandell, "Modeling of Marine Rope Fatigue Behavior," Text. Res. J., vol. 57, no. 6, pp. 318330, Jun. 1987.

[18] M. C. Kenney, J. F. Mandell, and F. J. McGarry, "Fatigue behaviour of synthetic fibres, yarns, and ropes," J. Mater. Sci., vol. 20, no. 6, pp. 2045-2059, Jun. 1985.

[19] M. Karayaka, S. Srinivasan, and S. S. Wang, "Advanced Design Methodology for Synthetic Moorings," presented at the Offshore Technology Conference, 1999.

[20] R. Tornqvist, M. Strande, D. Cannell, P. Gledhill, P. Smeets, and J. Gilmore, "Deployment of Subsea Equipment: Qualification of Large Diameter Fibre Rope for Deepwater Construction Applications," presented at the Offshore Technology Conference, 2011.

[21] O. Vennemann, I. Frazer, and R. Tornqvist, "Extending the Use of Conventional Construction Technology for the Installation of Subsea Production Facilities in Deep Water," presented at the Offshore Technology Conference, 2008. 
[22] M. Meuwissen, D. Glasbergen, M. Kösters, R. Bosman, P. Smeets, and H. Schneiders, "On the origin of lifetime extension for HMPE ropes in bending operations," in 2013 OCEANS - San Diego, 2013, pp. 1-10.

[23] P. Davies, Y. Reaud, L. Dussud, and P. Woerther, "Mechanical behaviour of HMPE and aramid fibre ropes for deep sea handling operations," Ocean Eng., vol. 38, no. 17-18, pp. 2208-2214, Dec. 2011.

[24] P. Davies, M. François, N. Lacotte, T. D. Vu, and D. Durville, "An empirical model to predict the lifetime of braided HMPE handling ropes under cyclic bend over sheave (CBOS) loading," Ocean Eng., vol. 97, pp. 74-81, 2015.

[25] B. D. Coleman, "Application of the theory of absolute reaction rates to the creep failure of polymeric filaments," J. Polym. Sci., vol. 20, no. 96, pp. 447-455, May 1956.

[26] R. P. M. Janssen, L. E. Govaert, and H. E. H. Meijer, "An Analytical Method To Predict Fatigue Life of Thermoplastics in Uniaxial Loading: Sensitivity to Wave Type, Frequency, and Stress Amplitude," Macromolecules, vol. 41, no. 7, pp. 2531-2540, Apr. 2008.

[27] L. E. Govaert, C. W. M. Bastiaansen, and P. J. R. Leblans, "Stress-strain analysis of oriented polyethylene," Polymer, vol. 34, no. 3, pp. 534-540, Jan. 1993.

[28] S. R. Ghoreishi, P. Cartraud, P. Davies, and T. Messager, "Analytical modeling of synthetic fiber ropes subjected to axial loads. Part I: A new continuum model for multilayered fibrous structures," Int. J. Solids Struct., vol. 44, no. 9, pp. 2924-2942, May 2007.

[29] S. R. Ghoreishi, P. Davies, P. Cartraud, and T. Messager, "Analytical modeling of synthetic fiber ropes. Part II: A linear elastic model for $1+6$ fibrous structures," Int. J. Solids Struct., vol. 44, no. 9, pp. 2943-2960, May 2007.

[30] T. D. Vu, D. Durville, and P. Davies, "Finite element simulation of the mechanical behavior of synthetic braided ropes and validation on a tensile test," Int. J. Solids Struct., vol. 58, pp. 106-116, Apr. 2015.

[31] W. X. Boleij, T. Engels, and L. Govaert, "Dynamic load performance of Dyneema," DSM, Geleen, Internal at DSM, 2015.

[32] A. R. Bunsell, Handbook of Tensile Properties of Textile and Technical Fibres. Elsevier, 2009. 


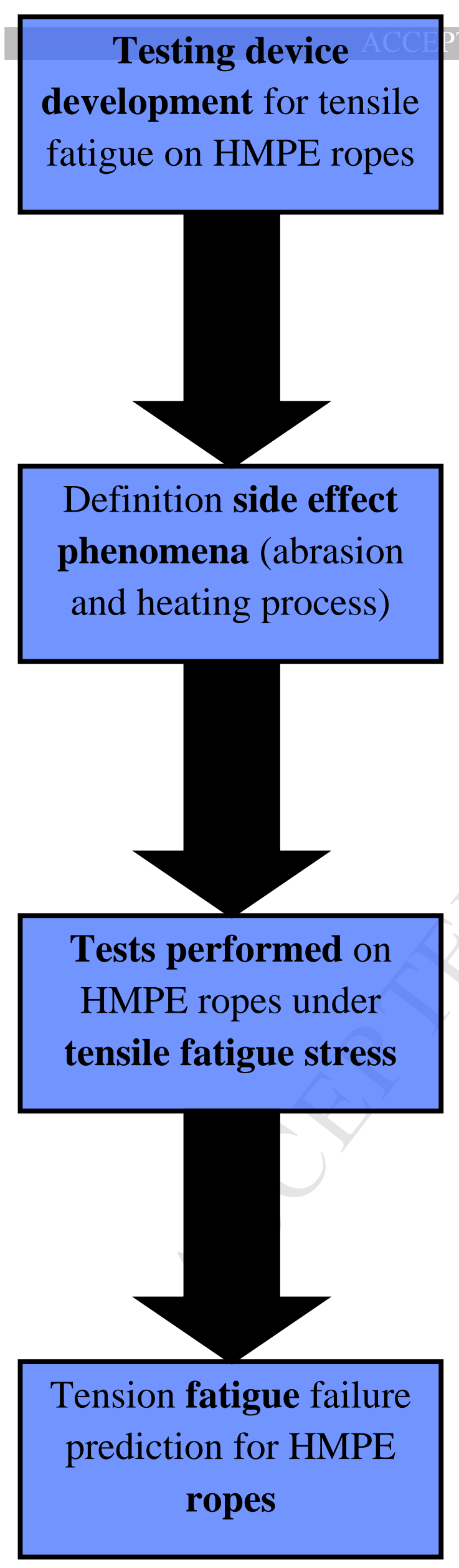

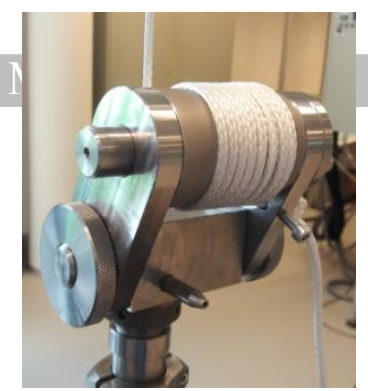

Clamping device

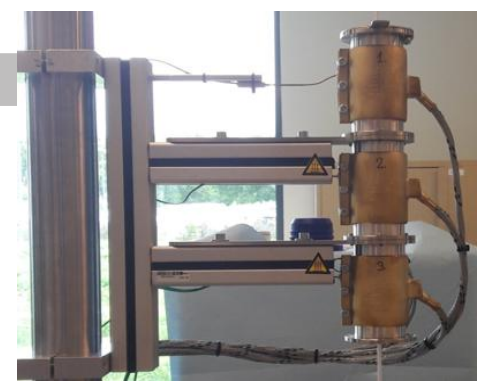

Oven for rope testing

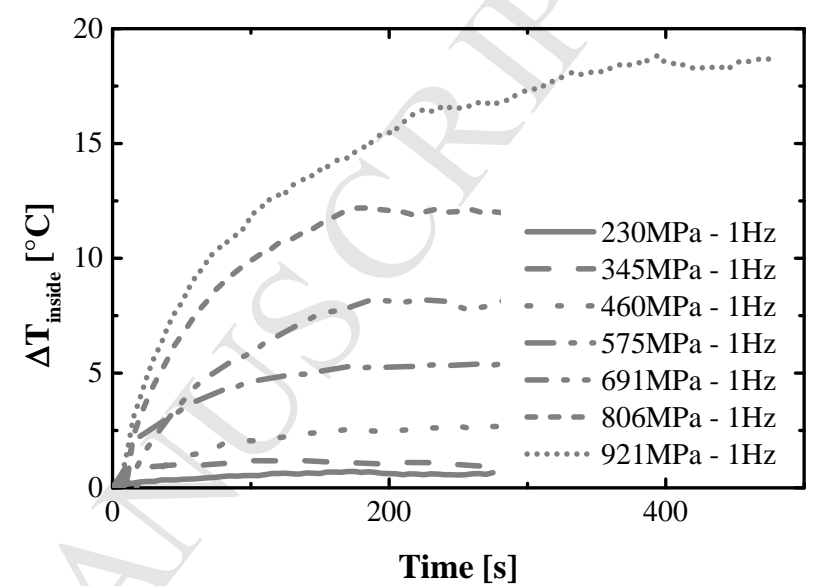

Temperature rise inside ropes for different loadings

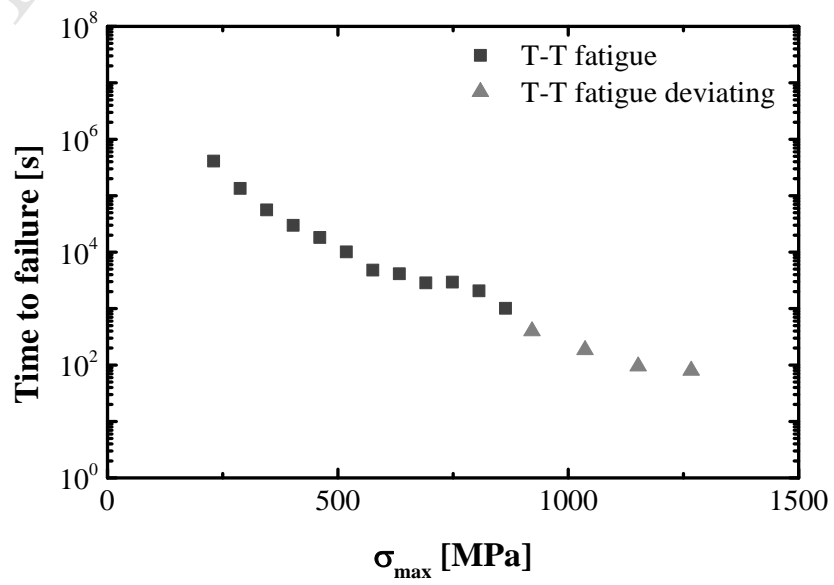

Experimental time to failure at $100^{\circ} \mathrm{C} R=0.1$ and $f=0.1 \mathrm{~Hz}$

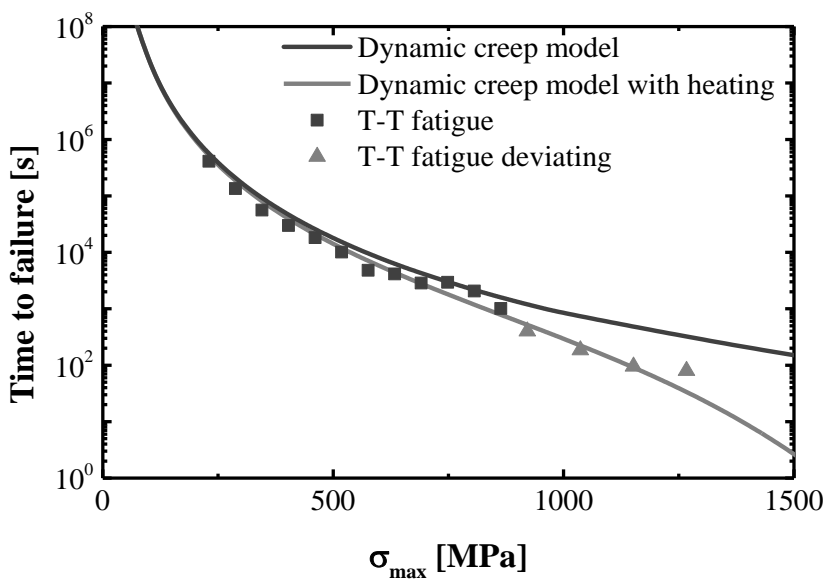

Comparison between experiments and theory 